\title{
Detection of apoptotic cells using a synthetic fluorescent sensor for membrane surfaces that contain phosphatidylserine
}

\author{
Cell Death and Differentiation (2003) 10, 1357-1359. doi:10.1038/sj.cdd.4401315 \\ Published online 5 September 2003
}

\section{Dear Editor,}

The appearance of phosphatidylserine (PS) on the cell surface is one of the hallmarks of the early/intermediate stages of cell apoptosis, and is believed to occur prior to DNA fragmentation, morphological changes, and plasma membrane permeabilization. ${ }^{1}$ The clearest assessment of cell apoptosis is gained from microscopy or flow cytometry analyses that examine large cell populations and identify and quantify subpopulations. A common method of detecting PS on a cell surface is to use the PS-binding protein annexin $V^{2,3}$ This $35 \mathrm{kDa}$ protein is typically labeled with a fluorescent dye, often fluorescein because it can be conveniently excited by an argon laser. Although annexin $\mathrm{V}$ is a useful reagent, it is an expensive protein, and there are some technical drawbacks. One concern is that up to $2.5 \mathrm{mM}$ of extracellular $\mathrm{Ca}^{2+}$ may be needed for complete membrane binding. This can lead to false-positive results because most animal cells have a $\mathrm{Ca}^{2+}$-dependent scramblase that can move PS to the cell surface. In addition, annexin $\mathrm{V}$ is known to associate with membrane surfaces that contain the by-products of lipid peroxidation. ${ }^{4}$ Furthermore, complete annexin binding requires incubations times of up to $1 \mathrm{~h}$, which is problematic for kinetic assays. ${ }^{5}$ In summary, dye-labeled annexin $\mathrm{V}$ is used extensively as a PS-sensing reagent, but there is a need for a low-molecular-weight mimic that can sense PS-rich membranes rapidly and independently of $\mathrm{Ca}^{2+}$ levels.

Inspection of an X-ray structure of annexin V bound to glycerophosphoserine shows that the phosphoserine head group can associate with one of the protein's four binding domains via two bridging calcium cations. ${ }^{6}$ This binding mechanism raises the possibility that rationally designed coordination compounds containing two or more appropriately spaced metal dications may act as functional mimics of annexin V. Here, we report on the membrane-binding and fluorescence-sensing properties of PSS-380, an anthracene derivative with two zinc dipicolylamine groups (Figure 1a). PSS-380 was recently shown to bind phosphorylated compounds like phosphotyrosine in water with association constants around $10^{7} \mathrm{M}^{-1}$. The strong association is due to the dianionic phosphate group coordinating simultaneously with the two zinc cations. These results suggested to us that PSS-380 may associate more strongly to the head groups of anionic phospholipids than to the head groups of zwitterionic phospholipids. In this letter, we show that PSS-380 can sense the presence of anionic phospholipids, especially PS, on the surface of vesicles and cells. In addition, we demonstrate the ability of PSS-380 to act as a fluorescent sensor of apoptotic cells.

The fluorescence emission of PSS-380 is unchanged if it is treated with zwitterionic phosphatidylcholine vesicles; however, there is a 10-fold increase in emission intensity upon the addition of anionic vesicles composed of $50: 50$ 1-palmitoyl-2oleoyl-sn-glycero-3-phosphocholine : 1-palmitoyl-2-oleoyl-snglycero-3-phosphoserine (POPC : POPS), and even vesicles containing $5 \mathrm{~mol} \%$ of POPS produce a four-fold rise in fluorescence emission (Figure 1b). Both of the zinc dipicolylamine groups in PSS-380 are necessary for the efficient detection of PS-enriched vesicles because treatment of AZD, a control analogue that lacks one of the zinc dipicolylamine groups, with excess POPC : POPS (50:50) vesicles produces very little enhancement in fluorescence.

We evaluated the ability of PSS-380 to detect cell apoptosis in three ways. First, we assessed whether PSS-380 could be used in a bulk fluorescence assay for apoptosis. We found that PSS-380 emission was on an average twice as intense $( \pm 5 \%)$ in the presence of apoptotic Jurkat cells (apoptosis induced by the anticancer drug camptothecin) than in the presence of an equal number of live cells. Thus, cell apoptosis can be detected qualitatively using PSS-380 in a simple, single-well, bulk fluorescence assay. We next used fluorescence microscopy to evaluate if PSS-380 could identify live, apoptotic, and dead cells. Cell apoptosis was induced by treatment of various cell types (Jurkat, MCF7, HEK293, and HL60) with camptothecin or the calcium ionophore A23187. The drug-treated cells and -untreated control cells were subsequently stained with a mixture of annexin V-FITC (green), PSS-380 (blue), and 7-aminoactinomycin D (7AAD) (red). Figure 1c displays typical microscopic images produced using this method. Live cells are not stained by any of the dyes, whereas staining by 7AAD identifies dead cells with permeable membranes. Cells that are only stained by annexin V-FITC or PSS-380 are defined as apoptotic. Competition studies showed that PSS-380 can block annexin V-FITC binding, which is evidence that they are competing for the same cell surface binding sites. The most compelling evidence that PSS-380 and annexin V are detecting the same population of apoptotic cells was gained from flow cytometry analysis of HL60 cells that were treated with camptothecin. The two sets of data in Figure 1d (UV excitation 
a
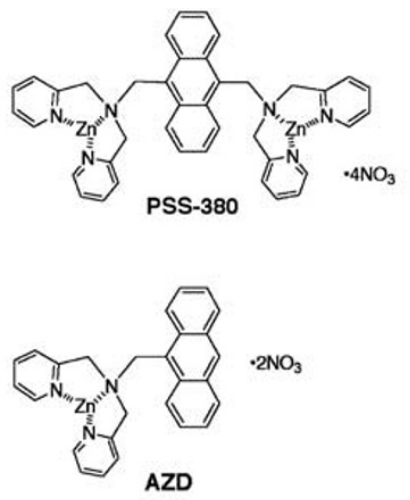

b

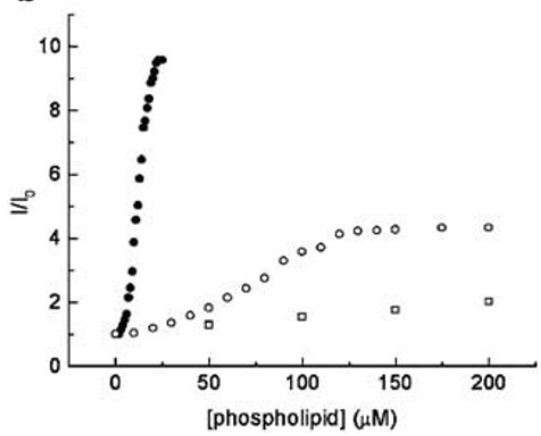

c
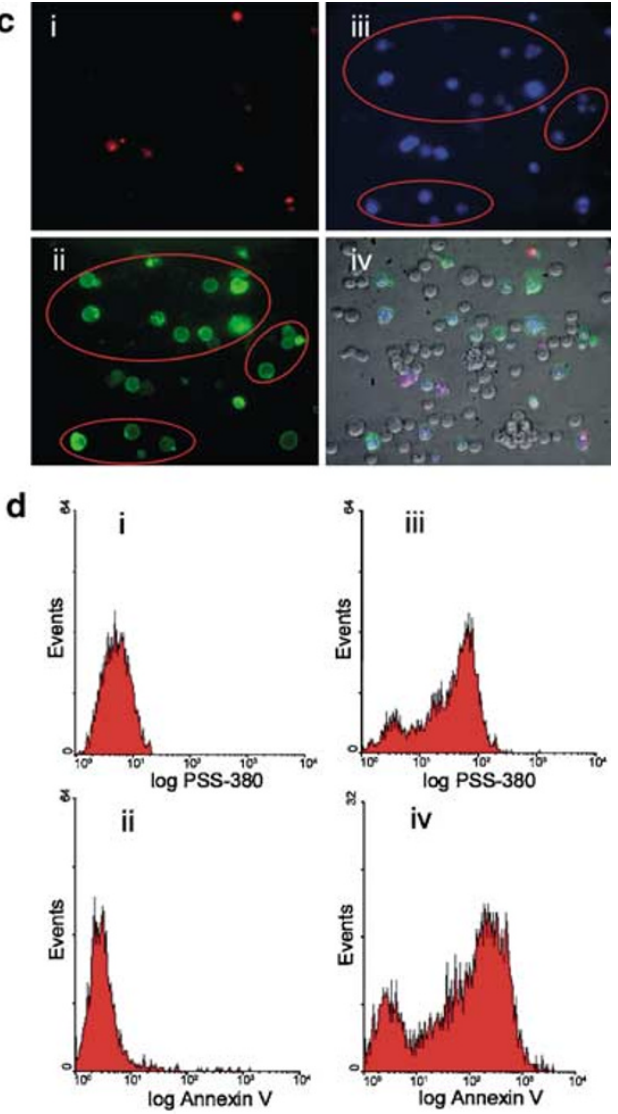

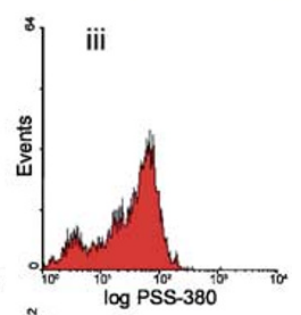

iv

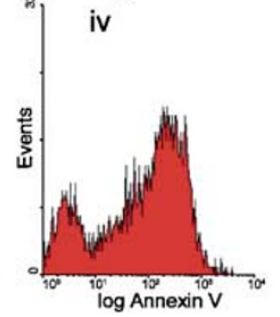

Figure 1 (a) Chemical structures of apoptosis sensor PSS-380 and control AZD. (b) Change in fluorescence intensity $I / I_{0}(e x 380$, em $440 \mathrm{~nm})$ of PSS-380 $(1 \mu \mathrm{M})$ in HEPES buffer (10 mM, pH 7.2) upon addition of $100 \mathrm{~nm}$ unilamellar vesicles composed of POPC : POPS (50:50, filled circles), POPC : POPS (95:5, open circles), $100 \%$ POPC (squares). (c) Apoptosis of Jurkat cells induced by incubation with camptothecin $(10 \mu \mathrm{M})$ for $4 \mathrm{~h}$. The cells were stained simultaneously with $7 \mathrm{AAD}(250 \mathrm{ng} /$ $\mathrm{ml}$ ) (i), annexinV-FITC (10 $\mu$ l of Pharmingen stock) (ii), and PSS-380 (20 $\mu \mathrm{M})$ (iii). Image iv is a merge of $\mathrm{i}-$ iii, and a phase-contrast image of the same cells. Live cells are not stained by any of the dyes, and apoptotic cells are highlighted by red ovals. (d) Flow cytometry analysis showing that treatment of HL60 cells with camptothecin $(10 \mu \mathrm{M})$ for $6 \mathrm{~h}$ induces cell apoptosis. Panels $\mathrm{i}$ and ii are untreated cells labeled with PSS-380 (ex 350, em $440 \mathrm{~nm}$ ) and annexin V-FITC (ex 488, em 525 nm). Panels iii and iv identify the cells that become apoptotic. All data shown are PI-negative cells

of PSS-380 and visible excitation of annexin V-FITC) lead to the same conclusion that $80-85 \%$ of the viable cells (identified because they are not stained by the vital cell dye propidium iodide $(\mathrm{PI})$ ) are apoptotic.

PSS-380 binding to apoptotic cells is almost instantaneous, and is not noticeably inhibited by the presence of $\mathrm{Ca}^{2+}$. Furthermore, PSS-380 staining fades rapidly when the mixture is treated with $10 \mathrm{mM}$ EDTA, confirming the critical role of the zinc atoms for cell binding, and also showing that the PSS-380 is not internalized by the cells. Tests were performed to establish the toxicity of PSS-380. Flow cytometry showed that exposure of Jurkat cells to $100 \mu \mathrm{M}$ of PSS-380 for $1 \mathrm{~h}$ produced no change in cell viability, as judged by the lack of staining by $\mathrm{PI}$.

In summary, the synthetic sensor PSS-380 appears to provide the same apoptosis detection information as annexin $\mathrm{V}$, but it is easier to handle and store. Not only should PSS380 be useful as an apoptosis sensor in cell biology research, it may also have utility in high-throughput assays for drug discovery, as well as automated monitoring of animal cell culture technology. ${ }^{3}$ A potential technical drawback with PSS380 is that it requires UV excitation, a feature that is presently not available on common flow cytometers. Our current research efforts are focused on making analogues of PSS380 that have altered fluorescence properties. Low-molecular-weight annexin mimics may also have potential as apoptotic tissue imaging agents. ${ }^{8}$

\section{Acknowledgements}

This study was funded by the National Institutes of Health, the Department of Defense, and the University of Notre Dame. We thank Professor Holly Goodson, and members of the Keck Center for Transgene Research for access to instrumentation. Researchers who wish to obtain a sample of PSS-380 should contact the corresponding author.

\section{AV Koulov ${ }^{1}$, KA Stucker $^{1}$, CLakshmi ${ }^{1}$, JP Robinson ${ }^{2}$ and BD Smith ${ }^{\star 1}$}

1 Department of Chemistry and Biochemistry, Walther Center for Cancer Research, University of Notre Dame, Notre Dame, IN 46556, USA

2 Purdue University Cytometry Laboratory, 201 South University Drive, Purdue University, West Lafayette, IN 47907, USA

* Corresponding author: BD Smith, Department of Chemistry and Biochemistry, Walther Center for Cancer Research, University of Notre Dame, Notre Dame, IN 46556, USA. Tel: 574-631-8632; Fax: 574-631-6652;

E-mail: smith.115@nd.edu 
1. Schlegel RA and Williamson $P$ (2001) Cell Death Differ. 8: 551-563

2. van Heerde WL, Robert-Offerman S, Dumont E, Hofstra L, Doevendans PA, Smits JF, Daemen MJ and Reutelingsperger CP (2000) Cardiovasc. Res. 45: 549-559

3. Pläsier B, Lloyd DR, Paul GC, Thomas CR and Al-Rubeai M (1999) J. Immunol. Methods 229: 81-95

4. Balasubramanian K, Bevers EM, Willems GM and Scroit AJ (2001) Biochemistry 40: $8672-8676$
5. Zweifach A (2000) Biochem. J. 349: 255-260

6. Sairjo MA, Concha NO, Kaetzel MA, Dedman JR and Seaton BA (1995) Nat. Struct. Biol. 2: 968-974

7. Ojida A, Mito-Oka Y, Inoue MA and Hamachi I (2002) J. Am. Chem. Soc. 124: 6256-6258

8. Blankenburg FG and Strauss HW (2001) Apoptosis 6: 117-123 